\title{
Semilocal E-preinvexity and its applications in nonlinear multiple objective fractional programming
}

\author{
Hehua Jiao ${ }^{1,2^{*}}$ and Sanyang Liu ${ }^{1}$
}

\author{
* Correspondence: jiaohh361@126. \\ com \\ 'Department of Mathematics, \\ Xidian University, Xi'an 710071, \\ China \\ Full list of author information is \\ available at the end of the article
}

\begin{abstract}
Introduction: In this paper, a new class of functions called semilocal E-preinvex functions is introduced, which is generalization of semi-E-preinvex functions and semilocal E-convex functions. Some of its basic properties are obtained.
\end{abstract}

Methods and Materials: Using $E-\eta$-semidifferentiability, some optimality conditions and duality results are established for a nonlinear multiobjective fractional programming with semilocal E-preinvex and related functions.

Conclusion: The results presented in this paper extend and generalize previously known results in this area.

Mathematics Subject Classification (2000): 90C26; 90C30; 90C46

Keywords: local E-invex set, semilocal E-preinvexity, multiobjective fractional programming, optimality, duality

\section{Introduction}

Convexity and generalized convexity play a vital role in the study of optimality and duality aspects of mathematical programming. Attempts have been made to generalize convexity to study their role in solving such types of problems. Generalizations of convexity related to optimality and duality for nonlinear singleobjective or multiobjective optimization problems have been of much interest in the recent past, and many contributions have been made to this development. See, e.g., [1-6] and the references therein.

After Ewing [7] presented the definition of semilocal convexity, by using more general semilocal preinvexity and $\eta$-semidifferentiability, Preda and Stancu-Minasian [8] gave optimality conditions for weak vector minima and extended the Wolfe and Mond-Weir duals, generalizing results of Preda and Stancu-Minasian [9]. Based on the results of [8,9], Preda further established optimality conditions and duality results for a nonlinear fractional multiple objective programming problem with semilocal preinvex functions involving $\eta$-semidifferentiability in [10].

On the other hand, Youness [11] proposed the concepts of $E$-convex sets, $E$-convex functions, and $E$-convex programming, discussed some of their basic properties, and obtained some optimality results on E-convex programming. Subsequently, Chen [12] brought forward a class of semi- $E$-convex functions and also discussed its basic properties. Moreover, by combining the concepts of semi-E-convexity and semilocal

(c) 2011 Jiao and Liu; licensee Springer. This is an Open Access article distributed under the terms of the Creative Commons Attribution License (http://creativecommons.org/licenses/by/2.0), which permits unrestricted use, distribution, and reproduction in any medium, provided the original work is properly cited. 
convexity, Hu et al. [13] put forward the concept of semilocal E-convexity, studied its some characterizations, and established some optimality conditions and duality results for semilocal E-convex programming. In [14], optimality and duality were further studied for a fractional multiple objective programming involving semilocal $E$-convexity. Recently, Fulga and Preda [15] extended the E-convexity to $E$-preinvexity and local $E$-preinvexity and studied some of their properties and an application. More recently, Luo and Jian [16] presented semi-E-preinvex maps in Banach spaces and discussed their properties.

Motivated by research works of [13-16] and references therein, in this paper, we introduce the concept of semilocal E-preinvexity and study some of its important properties. Then, we establish optimality conditions for a nonlinear fractional multiple objective programming problem (VFP) involving weaker $E$ - $\eta$-semidifferentiable functions. Finally, a general Bector type dual for (VFP) is formulated, and duality results are obtained using semilocal $E$-preinvex and related functions. The concept of semilocal $E$-preinvexity unifies the concepts of semilocal $E$-convexity and semi- $E$-preinvexity. Thus, we extend the work of $[10,13,14]$ and generalize results obtained in the literatures on this topic.

\section{Notations and definitions}

Throughout the paper, the convention below will be followed.

Let $R^{n}$ denote the $n$-dimensional Euclidean space, $E: R^{n} \rightarrow R^{n}$ and $\eta: R^{n} \times R^{n} \rightarrow R^{n}$ be two fixed mappings.

For any $x, y \in R^{n}$, by $x \leqq y$, we mean $x_{i} \leqq y_{i}$ for all $i$, by $x \leq y$, we mean $x_{i} \leqq y_{i}$ for all $i$ and $x_{j}<y_{j}$ for at least one $j \in\{1,2, \ldots, n\}$, by $x<y$, we mean $x_{i}<y_{i}$ for all $i$, and by $x \leqslant y$, we mean the negation of $x \leq y$.

In this section, we review some related definitions that will be used in the sequel.

Definition 2.1. [15] $A$ set $K \subset R^{n}$ is said to be E-invex with respect to $\eta$ if

$$
E(\gamma)+\lambda \eta(E(x), \quad E(\gamma)) \in K, \quad \forall x, y \in K, \lambda \in[0,1] .
$$

Definition 2.2. [16] Let $K \subset R^{n}$ be an E-invex set with respect to $\eta$. A function $f: R^{n} \rightarrow R$ is said to be semi-E-preinvex on $K$ with respect to $\eta$ if

$$
f(E(y)+\lambda \eta(E(x), \quad E(y))) \leqq \lambda f(x)+(1-\lambda) f(y), \quad \forall x, y \in K, \lambda \in[0,1] .
$$

Definition 2.3. [13]A set $K \subset R^{n}$ is said to be local starshaped E-convex, if there is a map $E$ such that corresponding to each pair of points $x, y \in K$, there is a maximal positive number $a(x, y) \leqq 1$ satisfying

$$
\lambda E(x)+(1-\lambda) E(y) \in K, \quad \forall \lambda \in[0, a(x, y)] .
$$

Definition 2.4. [13] A function $f: R^{n} \rightarrow R$ is said to be semilocal E-convex on a local starshaped E-convex set $K \subset R^{n}$ if for each pair of $x, y \in K$ (with a maximal positive number $a(x, y) \leqq 1$ satisfying (2.1)), there exists a positive number $b(x, y) \leqq a(x, y)$ satisfying

$$
f(\lambda(E(x))+(1-\lambda) E(y)) \leqq \lambda f(x)+(1-\lambda) f(y), \quad \forall \lambda \in[0, b(x, y)] .
$$

Definition 2.5. [15]A set $k \subset R^{n}$ is said to be local E-invex with respect to $\eta$, if $\forall x$, $y \in K$, there exists $a(x, y) \in(0,1]$ such that, $\forall \lambda \in[0, a(x, y)]$, 


$$
E(y)+\lambda \eta(E(x), \quad E(\gamma)) \in K .
$$

Remark 2.1. Every local starshaped $E$-convex set is a local $E$-invex set with respect to $\eta$, where $\eta(x, y)=x-y, \forall x, y \in R^{n}$. Every $E$-invex set with respect to $\eta$ is a local $E$ invex set with respect to $\eta$, where $a(x, y)=1, \forall x, y \in R^{n}$. But their converses are not necessarily true.

Example 2.1. Let $K=[-4,-1) \cup[1,4]$,

$$
\begin{aligned}
& E(x)=\left\{\begin{array}{l}
x^{2}, \text { if }|x| \leqq 2, \\
-1, \text { if }|x|>2 .
\end{array}\right. \\
& \eta(x, y)= \begin{cases}x-y, & \text { if } x \geqq 0, y \geqq 0, \text { or } x \leqq 0, y \leqq 0, \\
-1-y, & \text { if } x>0, y \leqq 0, \text { or } x \geqq 0, y<0, \\
1-y, & \text { if } x<0, y \geqq 0, \text { or } x \leqq 0, y>0 .\end{cases}
\end{aligned}
$$

We can testify that $K$ is a local E-invex set with respect to $\eta$. However, when $x_{0}=1$, $y_{0}=3$, there is a $\lambda_{1} \in[0,1]$ such that $E\left(y_{0}\right)+\lambda_{1} \eta\left(E\left(x_{0}\right), E\left(y_{0}\right)\right)=-1 \notin K$, i.e., $K$ is not an E-invex set with respect to $\eta$.

Similarly, for any positive number $a \leqq 1$, there exists a sufficiently small positive number $\lambda_{2} \leqq$ a satisfying $\lambda_{2} E\left(x_{0}\right)+\left(1-\lambda_{2}\right) E\left(y_{0}\right)=-1+2 \lambda_{2} \notin K$, that is, $K$ is not a local starshaped E-convex set.

Definition 2.6. [15]A function $f: R^{n} \rightarrow R$ is said to be local E-preinvex on $k \subset R^{n}$ with respect to $\eta$ if for any $x, y \in K$ (with a maximal positive number $a(x, y) \leqq 1$ satisfying (2.2)), there exists $0<b(x, y) \leqq a(x, y)$ such that $K$ is a local E-invex set and

$$
f(E(\gamma)+\lambda \eta(E(x), \quad E(y))) \leqq \lambda f(E(x))+1(1-\lambda) f(E(y)), \quad \forall \lambda \in[0, b(x, y)] .
$$

Definition 2.7. [17]A point $\bar{x} \in$ Xis said to be an efficient solution for programming problem $(M P): \min F(x)=\left(F_{1}(x), \ldots, F_{p}(x)\right), x \in X$, if there exists no $x \in X$ such that $F_{i}(x)<F_{i}(\bar{x})$ and $F_{i}(x)<F_{i}(\bar{x})$ for some $i \in\{1,2, \ldots, p\}$. A point $\bar{x} \in X$ is said to be a weak efficient solution for $(M P)$, if there exists no $x \in X$ such that $F(x)<F(\bar{x})$.

\section{Semilocal E-preinvexity}

In the section, we introduce the concepts of semilocal $E$-preinvex and related functions and study some of their properties.

Definition 3.1. A function $f: R^{n} \rightarrow R$ is said to be semilocal E-preinvex on $k \subset R^{n}$ with respect to $\eta$ if for any $x, y \in K$ (with a maximal positive number $a(x, y) \leqq 1$ satisfying (2.2)), there exists $0<b(x, y) \leqq a(x, y)$ such that $K$ is a local E-invex set and

$$
f(E(\gamma)+\lambda \eta(E(x), E(\gamma))) \leqq \lambda f(x)+(1-\lambda) f(\gamma), \quad \forall \lambda \in[0, b(x, y)] .
$$

If

$$
f(E(\gamma)+\lambda \eta(E(x), E(y))) \geqq \lambda f(x)+(1-\lambda) f(\gamma), \quad \forall \lambda \in[0, b(x, y)],
$$

then $f$ is called as a semilocal E-preincave function on $k$.

If the inequality signs above are strict for any $x, y \in K$ and $x \neq y$, then $f$ is called as a strict semilocal E-preinvex (E-preincave) function.

Remark 3.1. Every semilocal $E$-convex function is a semilocal $E$-preinvex function, where $\eta(x, y)=x-y, \forall x, y \in R^{n}$. Every semi-E-preinvex function with respect to $\eta$ is a 
semilocal $E$-preinvex function, where $a(x, y)=b(x, y)=1, \forall x, y \in R^{n}$. But their converses are not necessarily true.

We give below an example of semilocal $E$-preinvex function, which is neither a semilocal $E$-convex function nor a semi- $E$-preinvex function.

Example 3.1. Let the map $E: R \rightarrow R$ be defined as

$$
E(x)=\left\{\begin{array}{l}
0, \text { if } x<0 \\
1, \text { if } 1<x \leqq 2 \\
x, \text { if } 0 \leqq x \leqq 1 \text { or } x>2
\end{array}\right.
$$

and the map $\eta: R \times R \rightarrow R$ be defined as

$$
\eta(x, y)=\left\{\begin{array}{lr}
0, & \text { if } x=y \\
1-x, & \text { if } x \neq y .
\end{array}\right.
$$

Obviously, $R$ is a local starshaped E-convex set and a local E-invex set with respect to $\eta$. Let $f: R \rightarrow R$ be defined as

$$
f(x)= \begin{cases}0, & \text { if } 1<x \leqq 2 \\ 1, & \text { if } x>2 \\ -x+1, & \text { if } 0 \leqq x \leqq 1 \\ -x+2, & \text { if } x<0\end{cases}
$$

We can prove that $f$ is semilocal E-preinvex on $R$ with respect to $\eta$. However, when $x_{0}$ $=2, y_{0}=3$, and for any $b \in(0,1]$, there exists a sufficiently small $\lambda_{0} \in(0, b]$ satisfying

$$
f\left(\lambda_{0} E\left(x_{0}\right)+\left(1-\lambda_{0}\right) E\left(y_{0}\right)\right)=f\left(3-2 \lambda_{0}\right)=1>1-\lambda_{0}=\lambda_{0} f\left(x_{0}\right)+\left(1-\lambda_{0}\right) f\left(y_{0}\right) .
$$

That is, $f(x)$ is not a semilocal E-convex function on $R$.

Similarly, taking $x_{1}=1, y_{1}=4$, we have

$$
f\left(E\left(y_{1}\right)+\lambda_{1} \eta\left(E\left(x_{1}\right), E\left(y_{1}\right)\right)\right)=f(4)=1>1-\lambda_{1}=\lambda_{1} f\left(x_{1}\right)+\left(1-\lambda_{1}\right) f\left(y_{1}\right),
$$

for some $\lambda_{1} \in[0,1]$.

Thus, $f(x)$ is not a semi-E-preinvex function on $R$ with respect to $\eta$.

Definition 3.2. A real-valued function $f$ defined on a local E-invex set $k \subset R^{n}$ is said to be quasi-semilocal E-preinvex (with respect to $\eta$ ) if for all $x, y \in K$ (with a maximal positive number $a(x, y) \leqq 1$ satisfying (2.2))satisfying $f(x) \leqq f(y)$, there is a positive number $b(x, y) \leqq a(x, y)$ such that

$$
f(E(y)+\lambda \eta(E(x), E(y))) \leqq f(y), \quad \forall \lambda \in[0, b(x, y)] .
$$

Definition 3.3. A real-valued function $f$ defined on a local E-invex set $K \subset R^{n}$ is said to be pseudo-semilocal E-preinvex (with respect to $\eta$ ) if for all $x, y \in K$ (with a maximal positive number $a(x, y) \leqq 1$ satisfying (2.2)) satisfying $f(x)<f(y)$, there are a positive number $b(x, y) \leqq a(x, y)$ and a positive number $c(x, y)$ such that

$$
f(E(\gamma)+\lambda \eta(E(x), E(y))) \leqq f(\gamma)-\lambda c(x, y), \quad \forall \lambda \in[0, b(x, y)] .
$$

Remark 3.2. Every semilocal $E$-preinvex function on a local $E$-invex set $K$ with respect to $\eta$ is both a quasi-semilocal $E$-preinvex function and a pseudo-semilocal $E$-preinvex function.

Definition 3.4. Let $f: K \rightarrow R$ be a function, where $K \subset R^{n}$ is a local E-invex set with respect to $\eta$. We say that $f$ is $E$ - $\eta$-semidifferentiable at $\bar{x} \in$ Kif $E(\bar{x})=\bar{x}$ and 


$$
\begin{gathered}
f^{+}(\bar{x} ; \eta(E(x), \bar{x}))=\lim _{\lambda \rightarrow 0^{+}} \frac{1}{\lambda}[f(\bar{x}+\lambda \eta(E(x), \bar{x}))-f(\bar{x})] \text {,exists for each } x \in K \text {, where } \\
f^{+}(\bar{x} ; \eta(E(x), \bar{x}))=\lim _{\lambda \rightarrow 0^{+}} \frac{1}{\lambda}[f(\bar{x}+\lambda \eta(E(x), \bar{x}))-f(\bar{x})],
\end{gathered}
$$

(the right derivative at $\bar{x}$ along the direction $\eta(E(x), \bar{x})$ ).

Remark 3.3. If $E$ is an identity map, the $E$ - $\eta$-semidifferentiability is the $\eta$-semidifferentiability notion [18]. If $E$ is an identity map and $\eta(x, \bar{x})=x-\bar{x}$, the $E-\eta$-semidifferentiability is the semidifferentiability notion. If a function is directionally differentiable, then it is semidifferentiable, but the converse is not true.

Using Definition 3.1-3.4, the following lemma is immediate.

Lemma 3.1. (i). Let $f$ be semilocal E-preinvex (E-preincave) and E- $\eta$-semidifferentiable at $\bar{x} \in K \subset R^{n}$, where $K$ is a local E-invex set with respect to $\eta$. Then,

$$
f(x)-f(\bar{x}) \geqq(\leqq) f^{+}(\bar{x} ; \eta(E(x), \bar{x})), \quad \forall x \in K .
$$

(ii). Let $f$ be quasi(pseudo)-semilocal E-preinvex and E- $\eta$-semidifferentiable at $\bar{x} \in K \subset R^{n}$, where $K$ is a local E-invex set with respect to $\eta$. Then,

$$
f(x) \leqq(<) f(\bar{x}) \Rightarrow f^{+}(\bar{x} ; \eta(E(x), \bar{x})) \leqq(<) 0, \quad \forall x \in K .
$$

Theorem 3.1. Let $f: K \subset R^{n} \rightarrow R$ be a local E-preinvex function on a local E-invex set $K$ with respect to $\eta$, then $f$ is a semilocal E-preinvex function if and only if $f(E(x)) \leqq f(x)$, $\forall x \in K$.

Proof. Suppose that $f$ is a semilocal $E$-preinvex function on set $K$ with respect to $\eta$, then for each pair of points $x, y \in K$ (with a maximal positive number $a(x, y) \leqq 1$ satisfying (2.2)), there exists a positive number $b(x, y) \leqq a(x, y)$ satisfying

$$
f(E(x)+\lambda \eta(E(y), E(x))) \leqq \lambda f(y)+(1-\lambda) f(x), \quad \lambda \in[0, b(x, y)] .
$$

By letting $\lambda=0$, we have $f(E(x)) \leqq f(x), \forall x \in K$.

Conversely, assume that $f$ is a local $E$-preinvex function on a local $E$-invex set $K$, then for any $x, y \in K$, there exist $a(x, y) \in(0,1]$ satisfying $(2.2)$ and $b(x, y) \in(0, a(x, y)]$ such that

$$
f(E(\gamma)+\lambda \eta(E(x), E(y))) \leqq \lambda f(E(x))+(1-\lambda) f(E(y)), \quad \forall \lambda \in[0, b(x, y)] .
$$

Since $f(E(x)) \leqq f(x), \forall x \leqq K$, then

$$
f(E(y)+\lambda \eta(E(x), E(y))) \leqq \lambda f(x)+(1-\lambda) f(y), \quad \forall \lambda \in[0, b(x, y)] .
$$

The proof is completed.

Remark 3.4. A local $E$-preinvex function on a local $E$-invex set with respect to $\eta$ is not necessarily a semilocal $E$-preinvex function.

Example 3.2. Let $K=[-4,-1) \cup[1,4]$,

$$
\begin{aligned}
& E(x)=\left\{\begin{array}{l}
x^{2}, \text { if }|x| \leqq 2, \\
-1, \text { if }|x|>2,
\end{array}\right. \\
& \eta(x, y)= \begin{cases}x-y, & \text { if } x \geqq 0, y \geqq 0, \text { or } x \leqq 0, y \leqq 0, \\
-1-y, \text { if } x>0, y \leqq 0, \text { or } x \geqq 0, y<0, & \text { if } x<0, y \geqq 0, \text { or } x \leqq 0, y>0, \\
1-y,\end{cases}
\end{aligned}
$$

and $f: R \rightarrow R$ be defined by $f(x)=x^{2}$, then $f$ is local E-preinvex on $K$ with respect to $\eta$. 
Since $f(E(2))=16>f(2)=4$, from Theorem 3.1, it follows that $f$ is not a semilocal E-preinvex function.

Definition 3.5. The set $G=\left\{(x, \alpha): x \in K \subset R^{n}, \alpha \in R\right\}$ is said to be a local E-invex set with respect to $\eta$ corresponding to $R^{n}$ if there are two maps $\eta$, E and a maximal positive number $a\left(\left(x, \alpha_{1}\right),\left(y, \alpha_{2}\right)\right) \leqq 1$, for each $\left(x, \alpha_{1}\right),\left(y, \alpha_{2}\right) \in G$ such that

$$
\left(E(y)+\lambda \eta(E(x), E(y)), \lambda \alpha_{1}+(1-\lambda) \alpha_{2}\right) \in G, \quad \forall \lambda \in\left[0, a\left(\left(x, \alpha_{1}\right),\left(y, \alpha_{2}\right)\right)\right] .
$$

Theorem 3.2. Let $K \subset R^{n}$ be a local E-invex set with respect to $\eta$. Then $f$ is a semilocal E-preinvex function on $K$ with respect to $\eta$ if and only if its epigraph $G_{f}=\{(x, \alpha): x$ $\in K, f(x) \leqq \alpha, \alpha \in R\}$ is a local E-invex set with respect to $\eta$ corresponding to $R^{n}$.

Proof. Assume that $f$ is semilocal $E$-preinvex on $K$ with respect to $\eta$ and $\left(x, \alpha_{1}\right)$, $\left(y, \alpha_{2}\right) \in G_{f}$, then $x, y \in K, f(x) \leqq \alpha_{1}, f(y) \leqq \alpha_{2}$. Since $K$ is a local $E$-invex set, there is a maximal positive number $a(x, y) \leqq 1$ such that

$$
E(\gamma)+\lambda \eta(E(x), E(\gamma)) \in K, \quad \forall \lambda \in[0, a(x, y)] .
$$

In addition, in view of $f$ being a semilocal $E$-preinvex function on $K$ with respect to $\eta$, there is a positive number $b(x, y) \leqq a(x, y)$ such that

$$
f(E(y)+\lambda \eta(E(x), E(y))) \leqq \lambda f(x)+(1-\lambda) f(y) \leqq \lambda \alpha_{1}+(1-\lambda) \alpha_{2}, \quad \forall \lambda \in[0, b(x, y)] .
$$

That is, $\left(E(y)+\lambda \eta(E(x), E(y)), \lambda \alpha_{1}+(1-\lambda) \alpha_{2}\right) \in G_{f}, \forall \lambda \in[0, b(x, y)]$.

Therefore, $G_{f}=\{(x, \alpha): x \in K, f(x) \leqq \alpha, \alpha \in R\}$ is a local $E$-invex set with respect to $\eta$ corresponding to $R^{n}$.

Conversely, if $G_{f}$ is a local $E$-invex set with respect to $\eta$ corresponding to $R^{n}$, then for any points $(x, f(x)),(y, f(y)) \in G_{f}$, there is a maximal positive number $a((x, f(x))$, $(y, f(y))) \leqq 1$ such that

$$
(E(y)+\lambda \eta(E(x), E(y)), \lambda f(x)+(1-\lambda) f(y)) \in G_{f}, \quad \forall \lambda \in[0, a((x, f(x)),(y, f(y)))] .
$$

That is, $E(y)+\lambda \eta(E(x), E(y)) \in K$,

$$
f(E(y)+\lambda \eta(E(x), E(y))) \leqq \lambda f(x)+(1-\lambda) f(y), \quad \forall \lambda \in[0, a((x, f(x)),(y, f(y)))] .
$$

Thus, $K$ is a local $E$-invex set and $f$ is a semilocal $E$-preinvex function on $K$.

Theorem 3.3. If $f$ is a semilocal E-preinvex function on a local E-invex set $K \subset R^{n}$ with respect to $\eta$, then the level set $S_{\alpha}=\{x \in K: f(x) \leqq \alpha\}$ is a local E-invex set for any $\alpha \in R$.

Proof. For any $\alpha \in R$ and $x, y \in S_{\alpha}$, then $x, y \in K$ and $f(x) \leqq \alpha, f(y) \leqq \alpha$. Since $K$ is a local $E$-invex set, there is a maximal positive number $a(x, y) \leqq 1$ such that

$$
E(y)+\lambda \eta(E(x), E(y)) \in K, \forall \lambda \in[0, a(x, y)] .
$$

In addition, due to the semilocal $E$-preinvexity of $f$, there is a positive number $b(x, y)$ $\leqq a(x, y)$ such that

$$
f(E(y)+\lambda \eta(E(x), E(y))) \leqq \lambda f(x)+(1-\lambda) f(\gamma) \leqq \lambda \alpha+(1-\lambda) \alpha=\alpha, \quad \forall \lambda \in
$$
$[0, b(x, y)]$.

That is, $E(y)+\lambda \eta(E(x), E(y)) \in S_{\alpha}, \forall \lambda \in[0, b(x, y)]$.

Therefore, $S_{\alpha}$ is a local $E$-invex set with respect to $\eta$ for any $\alpha \in R$. 
Theorem 3.4. Let $f$ be a real-valued function defined on a local E-invex set $K \subset R^{n}$. Then $f$ is a semilocal E-preinvex function with respect to $\eta$ if and only if for each pair of points $x, y \in K$ (with a maximal positive number $a(x, y) \leqq 1$ satisfying (2.2)), there exists a positive number $b(x, y) \leqq a(x, y)$ such that

$$
f(E(y)+\lambda \eta(E(x), E(\gamma)))<\lambda \alpha+(1-\lambda) \beta, \quad \forall \lambda \in[0, b(x, y)],
$$

whenever $f(x)<\alpha, f(y)<\beta$.

Proof. Let $x, y \in K$ and $\alpha, \beta \in R$ such that $f(x)<\alpha, f(y)<\beta$. Due to the local $E$-invexity of $K$, there is a maximal positive number $a(x, y) \leqq 1$ such that

$$
E(\gamma)+\lambda \eta(E(x), E(\gamma)) \in K, \quad \forall \lambda \in[0, a(x, y)] .
$$

In addition, owing to the semilocal $E$-preinvexity of $f$, there is a positive number $b(x$, $y) \leqq a(x, y)$ such that

$$
f(E(y)+\lambda \eta(E(x), E(y))) \leqq \lambda f(x)+(1-\lambda) f(\gamma)<\lambda \alpha+(1-\lambda) \beta, \quad \forall \lambda \in[0, b(x, y)] .
$$

Conversely, let $(x, \alpha) \in G_{f},(y, \beta) \in G_{f}\left(\right.$ see epigraph $G_{f}$ in Theorem 3.2), then $x, y \in$ $K, f(x) \leqq \alpha, f(y) \leqq \beta$. Hence, $f(x)<\alpha+\epsilon$ and $f(y)<\beta+\epsilon$ hold for any $\epsilon>0$. According to the hypothesis, for $x, y \in K$ (with a positive number $a(x, y) \leqq 1$ satisfying (2.2)), there exists a positive number $b(x, y) \leqq a(x, y)$ such that

$$
f(E(y)+\lambda \eta(E(x), E(y)))<\lambda \alpha+(1-\lambda) \beta+\varepsilon, \quad \forall \lambda \in[0, b(x, y)] .
$$

Let $\epsilon \rightarrow 0^{+}$, then

$$
f(E(\gamma)+\lambda \eta(E(x), E(\gamma))) \leqq \lambda \alpha+(1-\lambda) \beta, \quad \forall \lambda \in[0, b(x, y)] .
$$

That is, $(E(y)+\lambda \eta(E(x), E(y)), \lambda \alpha+(1-\lambda) \beta) \in G_{f}, \forall \lambda \in[0, b(x, y)]$. Therefore, $G_{f}$ is a local $E$-invex set corresponding to $R^{n}$.

From Theorem 3.2, it follows that $f$ is semilocal $E$-preinvex on $K$ with respect to $\eta$.

\section{Optimality criteria}

In this section, we establish sufficient optimality conditions for fractional multiobjective programming with semilocal $E$-preinvex and related functions under $E$ - $\eta$-semidifferentiability assumptions.

We consider the following nonlinear fractional multiobjective programming problem:

$$
\text { (VFP) }\left\{\begin{array}{l}
\operatorname{minimize} \frac{f(x)}{g(x)}=\left(\frac{f_{1}(x)}{g_{1}(x)}, \ldots, \frac{f_{p}(x)}{g_{p}(x)}\right) \\
\text { subject to } h_{i}(x) \leqq 0, \quad j \in M=\{1,2, \ldots, m\} \\
x \in X_{0}
\end{array}\right.
$$

where $X_{0} \subseteq R^{n}$ is a local $E$-invex set and $g_{i}(x)>0$ for all $x \in X_{0}$ and each $i \in P=$ $\{1,2, \ldots, p\}$. Let $f=\left(f_{1}, f_{2}, \ldots, f_{p}\right), g=\left(g_{1}, g_{2}, \ldots, g_{p}\right)$ and $h=\left(h_{1}, h_{2}, \ldots, h_{m}\right)$, and denote $X=$ $\left\{x: h_{j}(x) \leqq 0, j \in M, x \in X_{0}\right\}$, the feasible set of problem (VFP). For $x^{*} \in X$, we put $M$ $\left(x^{*}\right)=\left\{j: h_{j}\left(x^{*}\right)=0, j \in M\right\}, N\left(x^{*}\right)=M \backslash M\left(x^{*}\right)$.

Utilizing the parametric approach of Jagannathan [19], we also formulate the nonlinear multi-objective programming problem as follows:

$$
\left(\mathrm{VFP}_{u}\right)\left\{\begin{array}{l}
\text { minimize }\left(f_{1}(x)-u_{1} g_{1}(x), \ldots, f_{p}(x)-u_{p} g_{p}(x)\right) \\
\text { subject to } h_{j}(x) \leqq 0, \quad j \in M, \\
x \in X_{0}
\end{array}\right.
$$


where $u=\left(u_{1}, u_{2}, \ldots, u_{p}\right) \in R^{p}$.

The following lemma connects the weak efficient solutions for (VFP) and (VFP $\left.{ }_{u}\right)$.

Lemma 4.1. A point $x^{*}$ is a weak efficient solution for (VFP) if and only if $x^{*}$ is a weak efficient solution for $\left(\mathrm{VFP}_{u}^{*}\right)$, where $u^{*}=\left(u_{1}^{*}, \ldots, u_{p}^{*}\right)=\left(\frac{f_{1}\left(x^{*}\right)}{g_{1}\left(x^{*}\right)}, \ldots, \frac{f_{p}\left(x^{*}\right)}{g_{p}\left(x^{*}\right)}\right)$.

Proof. Suppose that there exists a feasible point $x \in X$, such that for each $i \in P$,

$$
\begin{gathered}
f_{i}(x)-u_{i}^{*} g_{i}(x)<f_{i}\left(x^{*}\right)-u_{i}^{*} g_{i}\left(x^{*}\right) \\
\Rightarrow f_{i}(x)-u_{i}^{*} g_{i}(x)<f_{i}\left(x^{*}\right)-\frac{f_{i}\left(x^{*}\right)}{g_{i}\left(x^{*}\right)} g_{i}\left(x^{*}\right)=0 \\
\Rightarrow f_{i}(x)<\frac{f_{i}\left(x^{*}\right)}{g_{i}\left(x^{*}\right)} g_{i}(x) \Rightarrow \frac{f_{i}(x)}{g_{i}(x)}<\frac{f_{i}\left(x^{*}\right)}{g_{i}\left(x^{*}\right)},
\end{gathered}
$$

which contradicts the weak efficiency of $x^{*}$ for (VFP).

On the other hand, if there exists a feasible point $x \in X$ such that

$$
\frac{f_{i}(x)}{g_{i}(x)}<\frac{f_{i}\left(x^{*}\right)}{g_{i}\left(x^{*}\right)}=u_{i}^{*}
$$

then

$$
f_{i}(x)-u_{i}^{*} g_{i}(x)<0=f_{i}\left(x^{*}\right)-u_{i}^{*} g_{i}\left(x^{*}\right), \forall i \in P,
$$

and this is a contradiction to the weak efficiency of $x^{*}$ for $\left(\mathrm{VFP}_{u}{ }^{*}\right)$.

Thus, the proof is completed.

Now, we establish some sufficient optimality conditions for the problem (VFP).

Theorem 4.1. Let $\bar{x} \in X, E(\bar{x})=\bar{x}$, and $f$, $h$ be semilocal E-preinvex and $g$ be semilocal E-preincave, and they are all E- $\eta$-semidifferentiable at $\bar{x}$. Further, suppose that there are $\lambda^{0}=\left(\lambda_{1}^{0}, \lambda_{2}^{0}, \ldots, \lambda_{p}^{0}\right) \in R^{p}$, and $v^{0}=\left(v_{1}^{0}, v_{2}^{0}, \ldots, v_{m}^{0}\right) \in R^{m}$ such that

$$
\begin{aligned}
& \lambda^{0 T} f^{+}(\bar{x} ; \eta(E(x), \bar{x}))+v^{0 T} h^{+}(\bar{x} ; \eta(E(x), \bar{x})) \geqq 0, \quad \forall x \in X, \\
& g_{i}^{+}(\bar{x} ; \eta(E(x), \bar{x})) \leqq 0, \quad \forall x \in X, \quad i \in P, \\
& v^{0 T} h(\bar{x})=0, \\
& \lambda^{0} \geq 0, \quad v^{0} \geqq 0 .
\end{aligned}
$$

Then $\bar{x}$ is a weak efficient solution for (VFP).

Proof. By contradiction, suppose that $\bar{x}$ is not a weak efficient solution for (VFP), then there exists a point $\bar{x} \in X$ such that

$$
\frac{f_{i}(\hat{x})}{g_{i}(\hat{x})}<\frac{f_{i}(\bar{x})}{g_{i}(\bar{x})}, \quad i \in P .
$$

By the above hypotheses and Lemma 3.1, we have

$$
\begin{aligned}
& f_{i}(\hat{x})-f_{i}(\bar{x}) \geqq f_{i}^{+}(\bar{x} ; \eta(E(\hat{x}), \bar{x}), \quad i \in P, \\
& g_{i}(\hat{x})-g_{i}(\bar{x}) \leqq g_{i}^{+}(\bar{x} ; \eta(E(\hat{x}), \bar{x}), \quad i \in P, \\
& h_{j}(\hat{x})-h_{j}(\bar{x}) \geqq h_{j}^{+}(\bar{x} ; \eta(E(\hat{x}), \bar{x}), \quad j \in M .
\end{aligned}
$$


Multiplying (4.8) by $\lambda_{i}^{0}$, (4.10) by $v_{j}^{0}$, and summing the obtained relations, we get

$$
\sum_{i=1}^{p} \lambda_{i}^{0}\left(f_{i}(\hat{x})-f_{i}(\bar{x})\right)+\sum_{j=1}^{m} v_{j}^{0}\left(h_{j}(\hat{x})-h_{j}(\bar{x})\right) \geqq \lambda^{0 T} f^{+}(\bar{x} ; \eta(E(\hat{x}), \bar{x}))+v^{0 T} h^{+}(\bar{x} ; \eta(E(\hat{x}), \bar{x})) \geqq 0 .
$$

Since $\hat{x} \in X, v^{0} \geqq 0$, by (4.5) and (4.11), we further have

$$
\sum_{i=1}^{p} \lambda_{i}^{0}\left(f_{i}(\hat{x})-f_{i}(\bar{x})\right) \geqq 0
$$

Utilizing (4.6) and (4.12), we know that there exists at least an $i_{0}\left(1 \leqq i_{0} \leqq p\right)$ such that

$$
f_{i_{0}}(\hat{x}) \geqq f_{i_{0}}(\bar{x}) .
$$

On the other hand, (4.4) and (4.9) imply

$$
g_{i}(\hat{x}) \leqq g_{i}(\bar{x}), \quad i \in P .
$$

Now, using (4.13), (4.14), and $g>0$, we obtain

$$
\frac{f_{i_{0}}(\hat{x})}{g_{i_{0}}(\hat{x})} \geqq \frac{f_{i_{0}}(\bar{x})}{g_{i_{0}}(\bar{x})}
$$

which is in contradiction with (4.7).

Hence, the theorem is proved.

Similar to the proof of Theorem 4.1, we have the following theorem.

Theorem 4.2. Let $\bar{x} \in X, E(\bar{x})=\bar{x}$, and $f, g$, $h$ be E- $\eta$-semidifferentiable at $\bar{x}$. If there exist $\lambda^{0} \in R^{p}$ and $\nu^{0} \in R^{m}$ such that the conditions (4.3)-(4.6) hold and $\lambda^{0 T} f(x)+v^{0 T} h$ $(x)$ is a semilocal E-preinvex function, then $\bar{x}$ is a weak efficient solution for (VFP).

Theorem 4.3. Suppose that $\bar{x} \in X, E(\bar{x})=\bar{x}$, and $u_{i}^{0}=\frac{f_{i}(\bar{x})}{g_{i}(\hat{x})}(i \in P)$. Further suppose that $\left(f_{i}(x)-u_{i}^{0} g_{i}(x)\right)(i \in P)$ are all pseudo-semilocal E-preinvex functions and $h_{j}(x)(j \in M(\bar{x}))$ are all quasi-semilocal E-preinvex functions and $f, g$, $h$ are all E- $\eta$-semidifferentiable at $\bar{x}$. If there exist $\lambda^{0} \in R^{p}$ and $\nu^{0} \in R^{m}$ such that

$$
\begin{aligned}
& \sum_{i=1}^{p} \lambda_{i}^{0}\left(f_{i}^{+}(\bar{x} ; \eta(E(x), \bar{x}))-u_{i}^{0} g_{i}^{+}(\bar{x} ; \eta(E(x), \bar{x}))\right)+v^{0 T} h^{+}(\bar{x} ; \eta(E(x), \bar{x})) \geqq 0, \quad \forall x \in X, \\
& v^{0 T} h(\bar{x})=0, \\
& \lambda^{0} \geq 0, \quad v^{0} \geqq 0,
\end{aligned}
$$

then $\bar{x}$ is a weak efficient solution for (VFP).

Proof. We assume, by contradiction, that $\bar{x}$ is not a weak efficient solution for (VFP). Then, there exists $\breve{x} \in X$ such that

$$
\frac{f_{i}(\widetilde{x})}{g_{i}(\widetilde{x})}<\frac{f_{i}(\bar{x})}{g_{i}(\bar{x})}, \quad \text { i.e., } \quad f_{i}(\widetilde{x})-u_{i}^{0} g_{i}(\widetilde{x})<0, \quad i \in P,
$$

which means

$$
f_{i}(\breve{x})-u_{i}^{0} g_{i}(\breve{x})<f_{i}(\bar{x})-u_{i}^{0} g_{i}(\breve{x})<0, \quad i \in P .
$$


By the pseudo-semilocal $E$-preinvexity of $\left(f_{i}(x)-u_{i}^{0} g_{i}(x)\right)(i \in P)$ and Lemma 3.1, we have

$$
f_{i}^{+}(\bar{x} ; \eta(E(\bar{x}), \bar{x}))-u_{i}^{0} g_{i}^{+}(\bar{x} ; \eta(E(\bar{x}), \bar{x})<0, \quad i \in P .
$$

Utilizing $\lambda^{0} \geq 0$, we get

$$
\sum_{i=1}^{p} \lambda_{i}^{0}\left(f_{i}^{+}(\bar{x} ; \eta(E(\breve{x}), \bar{x}))-u_{i}^{0} g_{i}^{+}(\bar{x} ; \eta(E(\breve{x}), \bar{x}))\right)<0 .
$$

From $h(\breve{x}) \leqq 0$ and $h_{j}(\bar{x})=0, j \in M(\bar{x})$, we have $h_{j}(\breve{x}) \leqq h_{j}(\bar{x}), \forall j \in M(\bar{x})$. By the quasi-semilocal $E$-preinvexity of $h_{j}$ and Lemma 3.1, we obtain

$$
h_{j}^{+}(\bar{x} ; \eta(E(\bar{x}), \bar{x})) \leqq 0, \quad \forall j \in M(\bar{x}) .
$$

Considering $v^{0} \geqq 0$ and $v_{j}^{0}=0$ for $j \in N(\bar{x})$, one has

$$
\sum_{j=1}^{m} v_{j}^{0} h_{j}^{+}(\bar{x} ; \eta(E(\bar{x}), \bar{x})) \leqq 0
$$

Hence, by (4.19) and (4.20), we have

$$
\sum_{i=1}^{p} \lambda_{i}^{0}\left(f_{i}^{+}(\bar{x} ; \eta(E(\breve{x}), \bar{x}))-u_{i}^{0} g_{i}^{+}(\bar{x} ; \eta(E(\breve{x}), \bar{x}))\right)+\sum_{j=1}^{m} v_{j}^{0} h_{j}^{+}(\bar{x} ; \eta(E(\bar{x}), \bar{x}))<0,
$$

which is in contradiction with relation (4.16) at point $\breve{x} \in X$.

Therefore, $\bar{x}$ is a weak efficient solution for (VFP).

Analogously, we have the theorem below.

Theorem 4.4. Let $\bar{x} \in X, E(\bar{x})=\bar{x}, u_{i}^{0}=\frac{f_{i}(\bar{x})}{g_{i}(\bar{x})}(i \in P)$, and $f, g$, $h$ be E- $\eta$-semidifferentiable at $\bar{x}$. If there exist $\lambda^{0} \in R^{p}$ and $v^{0} \in R^{m}$ such that the conditions (4.16)-(4.18) hold and $\sum_{i=1}^{p} \lambda_{i}^{0}\left(f_{i}(x)-u_{i}^{0} g_{i}(x)\right)+v_{M(\bar{x})}^{0 T} h_{M(\bar{x})}(x)$ is a pseudo-semilocal E-preinvex function, then $\bar{x}$ is a weak efficient solution for (VFP).

The following corollary is followed directly by Theorem 4.3 or Theorem 4.4.

Corollary 4.1. Let $\bar{x} \in X, E(\bar{x})=\bar{x}$, and $u_{i}^{0}=\frac{f_{i}(\bar{x})}{g_{i}(\bar{x})}(i \in P)$. Further suppose that $f$, $f, h_{M(\bar{x})}$ are all semilocal E-preinvex functions, $g$ is a semilocal E-preincave function, and $f, g, h$ are all E- $\eta$-semidifferentiable at $\bar{x}$. If there exist $\lambda^{0} \in R^{p}$ and $v^{0} \in R^{m}$ such that the conditions (4.16)-(4.18) hold, then $\bar{x}$ is a weak efficient solution for (VFP).

\section{Duality}

Utilizing the approaches of Bector et al. [20], we formulate the dual problem for (VFP) as follows:

$$
\text { (VFD) } \begin{cases}\text { maximize } & \left(\lambda_{1}, \lambda_{2}, \ldots, \lambda_{p}\right) \\ \text { subject to } & \sum_{i=1}^{p} \alpha_{i}\left(f_{i}^{+}(u ; \eta(E(x), u))-\lambda_{i} g_{i}^{+}(u ; \eta(E(x), u))\right) \\ & +\sum_{j=1}^{m} \beta_{j} h_{j}^{+}(u ; \eta(E(x), u)) \geqq 0, \quad \forall x \in X_{0}, \\ f_{i}(u)-\lambda_{i} g_{i}(u) \geqq 0, & i \in P, \\ \beta_{j} h_{j}(u) \geqq 0, & j \in M, \\ \lambda=\left(\lambda_{1}, \ldots, \lambda_{p}\right) \geqq 0, & \alpha=\left(\alpha_{1}, \ldots, \alpha_{p}\right)>0, \\ \beta=\left(\beta_{1}, \ldots, \beta_{m}\right) \geqq 0, & u \in X_{0} .\end{cases}
$$


Denote the feasible set of problem (VFD) by $X^{\prime}$.

Theorem 5.1. (Weak duality) Let $x \in X,(u, \alpha, \beta, \lambda) \in X^{\prime}$, and $E(u)=u$. If $\sum_{i=1}^{p} \alpha_{i}\left(f_{i}-\lambda_{i} g_{i}\right)$ is a pseudo-semilocal E-preinvex function, and $\sum_{j=1}^{m} \beta_{j} h_{j} i s$ a quasisemilocal E-preinvex function, and they are all E- $\eta$-semidierentiable at $u$, then $\frac{f(x)}{g(x)} \nless \lambda$.

Proof. We proceed by contradicting. Then, if the conclusion is not true, we have

$$
\begin{gathered}
\frac{f(x)}{g(x)} \leq \lambda, \quad \text { i.e., } \quad f_{i}(x)-\lambda_{i} g_{i}(x) \leqq 0, \quad \forall i \in P, \\
f_{k}(x)-\lambda_{k} g_{k}(x)<0, \quad \text { for some } k \in P .
\end{gathered}
$$

From $\alpha>0$ and $(u, \alpha, \beta, \lambda) \in X^{\prime}$, we get

$$
\sum_{i=1}^{p} \alpha_{i}\left(f_{i}(x)-\lambda_{i} g_{i}(x)\right)<0 \leqq \sum_{i=1}^{p} \alpha_{i}\left(f_{i}(u)-\lambda_{i} g_{i}(u)\right) .
$$

By the pseudo-semilocal $E$-preinvexity of $\sum_{i=1}^{p} \alpha_{i}\left(f_{i}-\lambda_{i} g_{i}\right)$ and Lemma 3.1, we obtain

$$
\left(\sum_{i=1}^{p} \alpha_{i}\left(f_{i}-\lambda_{i} g_{i}\right)\right)^{+}(u ; \eta(E(x), u))<0,
$$

that is, $\sum_{i=1}^{p} \alpha_{i}\left(f_{i}^{+}(u ; \eta(E(x), u))-\lambda_{i} g_{i}^{+}(u ; \eta(E(x), u))\right)<0$.

On the other hand, from $\beta \geqq 0$ and $x \in X$, it follows that

$$
\sum_{j=1}^{m} \beta_{j} h_{j}(x) \leqq 0 \leqq \sum_{j=1}^{m} \beta_{j} h_{j}(u) .
$$

Using the quasi-semilocal $E$-preinvexity of $\sum_{j=1}^{m} \beta_{j} h_{j}$ and Lemma 3.1, one has

$$
\left(\sum_{j=1}^{m} \beta_{j} h_{j}\right)^{+}(u ; \eta(E(x), u)) \leqq 0, \quad \sum_{j=1}^{m} \beta_{j} h_{j}^{+}(u ; \eta(E(x), u)) \leqq 0 .
$$

Therefore,

$$
\sum_{i=1}^{p} \alpha_{i}\left(f_{i}^{+}(u ; \eta(E(x), u))-\lambda_{i} g_{i}^{+}(u ; \eta(E(x), u))\right)+\sum_{j=1}^{m} \beta_{j} h_{j}^{+}(u ; \eta(E(x), u))<0,
$$

which is in contradiction with $(u, \alpha, \beta, \lambda) \in X$.

Similarly, we have the following weak duality.

Theorem 5.2. (Weak duality) Let $x \in X,(u, \alpha, \beta, \lambda) \in X^{\prime}$, and $E(u)=u$. If $\sum_{i=1}^{p} \alpha_{i}\left(f_{i}-\lambda_{i} g_{i}\right)+\sum_{j=1}^{m} \beta_{j} h_{j}$ is pseudo-semilocal E-preinvex and E- $\eta$-semidifferentiable at $u$, then $\frac{f(x)}{g(x)} \nless \lambda$.

Theorem 5.3. (Converse duality) Let $\bar{x} \in X$ and $\left(x^{*}, \alpha^{*}, \beta^{*}, \lambda^{*}\right) \in X, E\left(x^{*}\right)=x^{*}$, where $\lambda^{*}=\frac{f\left(x^{*}\right)}{g\left(x^{*}\right)}=\frac{f(\bar{x})}{g(\bar{x})}=\left(\lambda_{1}^{*}, \ldots, \lambda_{p}^{*}\right)$. If $f_{i}-\lambda_{i}^{*} g_{i}(i \in P), h_{j}(j \in M)$ are all semilocal Epreinvex functions and all E- $\eta$-semidifferentiable at $x^{*}$, then $\bar{x}$ is a weak efficient solution for (VFP). 
Proof. By the given hypotheses and Lemma 3.1, for any $x \in X$, we get

$$
\begin{gathered}
\left(f_{i}(x)-\lambda_{i}^{*} g_{i}(x)\right)-\left(f_{i}\left(x^{*}\right)-\lambda_{i}^{*} g_{i}\left(x^{*}\right)\right) \geqq f_{i}^{+}\left(x^{*} ; \eta\left(E(x), x^{*}\right)\right)-\lambda_{i}^{*} g_{i}^{+}\left(x^{*} ; \eta\left(E(x), x^{*}\right)\right), \quad i \in P ; \\
h_{j}(x)-h_{j}\left(x^{*}\right) \geqq h_{j}^{+}\left(x^{*} ; \eta\left(E(x), x^{*}\right)\right), \quad j \in M .
\end{gathered}
$$

Utilizing the first constraint condition for (VFD), $\alpha^{*}>0, \beta^{*} \geqq 0, \lambda^{*} \geqq 0$ and the two inequalities above, we have

$$
\begin{gathered}
\sum_{i=1}^{p} \alpha_{i}^{*}\left(\left(f_{i}(x)-\lambda_{i}^{*} g_{i}(x)\right)-\left(f_{i}\left(x^{*}\right)-\lambda_{i}^{*} g_{i}\left(x^{*}\right)\right)\right)+\sum_{j=1}^{m} \beta_{j}^{*}\left(h_{j}(x)-h_{j}\left(x^{*}\right)\right) \geqq \\
\sum_{i=1}^{p} \alpha_{i}^{*}\left(f_{i}^{+}\left(x^{*} ; \eta\left(E(x), x^{*}\right)\right)-\lambda_{i}^{*} g_{i}^{+}\left(x^{*} ; \eta\left(E(x), x^{*}\right)\right)\right)+\sum_{j=1}^{m} \beta_{j}^{*} h_{j}^{+}\left(x^{*} ; \eta\left(E(x), x^{*}\right)\right) \geqq 0 .
\end{gathered}
$$

In view of $h_{j}(x) \leqq 0, \beta_{j}^{*} \geqq 0, \beta_{j}^{*} h_{j}\left(x^{*}\right) \geqq 0(j \in M)$ and $\lambda_{i}^{*}=\frac{f_{i}\left(x^{*}\right)}{g_{i}\left(x^{*}\right)}(i \in P)$, one has

$$
\sum_{i=1}^{p} \alpha_{i}^{*}\left(f_{i}(x)-\lambda_{i}^{*} g_{i}(x)\right) \geqq 0, \quad \forall x \in X .
$$

Now, we assume that $\bar{x}$ is not a weak efficient solution for (VFP). From $\lambda_{i}^{*}=\frac{f_{i}(\bar{x})}{g_{i}(\bar{x})}(i \in P)$ and Lemma 4.1, it follows that $\bar{x}$ is not a weak efficient solution for $\left(\operatorname{VFP}_{\lambda^{*}}\right)$. Hence, there exists an $\tilde{x} \in X$ such that $f_{i}(\tilde{x})-\lambda_{i}^{*} g_{i}(\tilde{x})<f_{i}(\bar{x})-\lambda_{i}^{*} g_{i}(\bar{x})=0$, $i \in P$, which implies that

$$
\sum_{i=1}^{p} \alpha_{i}^{*}\left(f_{i}(\tilde{x})-\lambda_{i}^{*} g_{i}(\tilde{x})\right)<0 .
$$

This is a contradiction to the inequality (5.2).

So the theorem is proved.

\section{Acknowledgements}

This work was supported by National Science Foundation of China, No.60974082.

\section{Author details}

${ }^{1}$ Department of Mathematics, Xidian University, Xi'an 710071, China ${ }^{2}$ College of Mathematics and Computer Science, Yangtze Normal University, Chongqing 408100, China

\section{Authors' contributions \\ $\mathrm{JHH}$ proposed the concept of semilocal E-preinvex functions and discussed some of its important properties. Additionally, $\mathrm{JHH}$ carried out the studies of optimality and duality for a nonlinear multiobjective fractional programming with semilocal E-preinvex and related functions and drafted the manuscript. LSY participated in the design, coordination and examination of this paper. All authors read and approved the final manuscript.}

\section{Competing interests}

The authors declare that they have no competing interests.

Received: 30 April 2011 Accepted: 22 November 2011 Published: 22 November 2011

\section{References}

1. Egudo, RR: Efficiency and generalized convex duality for multiobjective programs. J Math Anal Appl. 138, 84-94 (1989). doi:10.1016/0022-247X(89)90321-1

2. Geoffrion, AM: Proper efficiency and the theory of vector maximization. J Math Anal Appl. 22, 618-630 (1968). doi:10.1016/0022-247X(68)90201-1

3. Mishra, SK: On multiple-objective optimization with generalized univexity. J Math Anal Appl. 224, 131-148 (1998). doi:10.1006/jmaa.1998.5992

4. Mukherjee, RN, Mishra, SK: Multiobjective programming with semilocally convex functions. J Math Anal Appl. 199, 409-424 (1996). doi:10.1006/jmaa.1996.0150 
5. Rueda, NG, Hanson, MA: Optimality criteria in mathematical programming involving generalized invexity. J Math Anal Appl. 130, 375-385 (1988). doi:10.1016/0022-247X(88)90313-7

6. Yang, XM, Li, D: Semistrictly preinvex functions. J Math Anal Appl. 258, 287-308 (2001). doi:10.1006/jmaa.2000.7382

7. Ewing, GM: Sufficient conditions for global minima of suitably convex functions from variational and control theory. SIAM Rev. 19, 202-220 (1977). doi:10.1137/1019037

8. Preda, V, Stancu-Minasian, IM: Duality in multiple objective programming involving semilocally preinvex and related functions. Glas Mat Ser III. 32, 153-165 (1997)

9. Preda, V, Stancu-minasian, IM, Batatorescu, A: Optimality and duality in nonlinear programming involving semilocally preinvex and related functions. J Inf Optim Sci. 17, 585-596 (1996)

10. Preda, V: Optimality and duality in fractional multiple objective programming involving semilocally preinvex and related functions. J Math Anal Appl. 288, 365-382 (2003). doi:10.1016/S0022-247X(02)00460-2

11. Youness, EA: E-convex sets, E-convex functions and E-convex programming. J Op-tim Theory Appl. 102, 439-450 (1999)

12. Chen, XS: Some properties of semi-E-convex functions. J Math Anal Appl. 275, 251-262 (2002). doi:10.1016/S0022-247X (02)00325-6

13. Hu, QJ, Jian, JB, Zheng, HY, Tang, CM: Semilocal E-convexity and semilocal E-convex programming. Bull Aust Math Soc. 75, 59-74 (2007). doi:10.1017/S0004972700038983

14. $\mathrm{Hu}, \mathrm{QJ}, \mathrm{XiaO}, \mathrm{YH}, \mathrm{Chen}, \mathrm{NP}$ : Optimality and duality on fractional multi-objective programming under semilocal $E-$ convexity, Chin. Quart J Math. 24, 200-210 (2009)

15. Fulga, C, Preda, V: Nonlinear programming with E-preinvex and local E-preinvex functions. Eur J Oper Res. 192, 737-743 (2009). doi:10.1016/j.ejor.2007.11.056

16. Luo, ZM, Jian, JB: Some properties of semi-E-preinvex maps in Banach spaces, Nonlinear Anal. Real World Appl. 12, 1243-1249 (2011). doi:10.1016/j.nonrwa.2010.09.019

17. Chankong, V, Haimes, YY: Multiobjective Decision Making: Theory and Methodology. North-Holland, New York (1983)

18. Niculescu, C: Optimality and duality in multiobjective fractional programming involving $\rho$-semilocally type I-preinvex and related functions. J Math Anal Appl. 335, 7-19 (2007). doi:10.1016/j.jmaa.2006.12.084

19. Jagannathan, R: Duality for nonlinear fractional programms. Oper Res. 17, 1-3 (1973). doi:10.1007/BF01951364

20. Bector, CR, Chandra, S, Husain, I: Optimality conditions and duality in subdifferentiable multiobjective fractional programming. J Optim Theory Appl. 79, 105-125 (1993). doi:10.1007/BF00941889

doi:10.1186/1029-242X-2011-116

Cite this article as: Jiao and Liu: Semilocal E-preinvexity and its applications in nonlinear multiple objective fractional programming. Journal of Inequalities and Applications 2011 2011:116.

\section{Submit your manuscript to a SpringerOpen ${ }^{\circ}$ journal and benefit from:}

- Convenient online submission

- Rigorous peer review

- Immediate publication on acceptance

- Open access: articles freely available online

- High visibility within the field

- Retaining the copyright to your article

Submit your next manuscript at $\gg$ springeropen.com 